\title{
ON GROUPS OF AUTOMORPHISMS OF FINITE FACTORS
}

\author{
TEISHIRÔ SAITÔ
}

(Received June 7,1961)

Introduction. One of the important questions in the theory of the crossed products of rings of operators is the following: Is the crossed product of a finite factor $\mathbf{M}$ also a finite factor for any group $G$ of automorphisms of $\mathbf{M}$ ? The answer for this question is negative in general ([4]), and some kinds of conditions on $G$ under which the crossed product is a factor have been obtained ([4]). In $\S 2$ we shall deal with this question when $G$ is abelian, and sharpen the results in [2]. In $\$ 3$ we shall consider the behaviour of the action of $G$ in the crossed product and give a condition on $G$ under which the crossed product is a factor.

1. Throughout this paper, we assume that all $W^{*}$-algebras are finite factors with the invariants $C=1$. An automorphism of a $W^{*}$-algebra means a *automorphism, and a group of outer automorphisms of a $W^{*}$-algebra is a group of automorhisms all member of which are outer automorphisms except the unit. The unit of a group will be denoted by e. $R\left(a_{\lambda} \mid \lambda \in \Lambda\right)$ means the $W^{*}$-algebra generated by the family of operators $a_{\lambda}(\lambda \in \Lambda)$.

For convenience sake, we shall explain the construction of the crossed product. Let $\mathbf{M}$ be a finite factor with the invariant $C=1$ on a Hilbert space $\mathbf{H}$ and $G$ a group of automorphisms of $\mathbf{M}$. Let $\boldsymbol{\varphi}$ be a separating and generating trace vector for $\mathbf{M}$. For each $\sigma \in G$ we define

$$
u_{\sigma}(a \varphi)=a^{\sigma-1} \varphi \quad \text { for all } a \in \mathbf{M}
$$

where $a^{\tau}$ is the image of $a$ by an automorphism $\tau$. Then $u_{\sigma}$ can be extended to a unitary operator on $\mathbf{H}$ which will be also denoted by $u_{\sigma}$, and $\sigma \rightarrow u_{\sigma}$ is a faithful unitary representation of $G$ on $\mathbf{H}$ such that

$$
u_{\sigma}^{*} a u_{\sigma}=a^{\sigma} \quad \text { for all } a \in \mathbf{M} \text {. }
$$

Now consider the Hilbert space $\mathbf{H} \otimes l_{2}(G)$. If we choose the complete orthonormal system $\left\{\varepsilon_{\alpha}\right\}_{\alpha \in G}$ in $l_{2}(G)$ such as

$$
\varepsilon_{\alpha}(\gamma)= \begin{cases}1 & \text { if } \quad \gamma=\alpha \\ 0 & \text { otherwise, }\end{cases}
$$

each vector of $\mathbf{H} \otimes l_{2}(G)$ is expressed in the form $\sum \boldsymbol{\phi}_{\alpha} \otimes \varepsilon_{\alpha}$ where $\boldsymbol{\varphi}_{\alpha} \in \mathbf{H}$ 
and $\sum_{\alpha \in G}\left\|\boldsymbol{\varphi}_{\alpha}\right\|^{2}<\infty$.

For each $a \in \mathbf{M}$ and $\sigma \in G$ we define the operators $a \otimes 1$ and $U_{\sigma}$ on $\mathbf{H} \otimes l_{2}(G)$ by

$$
(a \otimes 1)\left(\sum_{\alpha \in G} \boldsymbol{\varphi}_{\alpha} \otimes \varepsilon_{\alpha}\right)=\sum_{\alpha \in G} a \varphi_{\alpha} \otimes \varepsilon_{\alpha}
$$

and

$$
U_{\sigma}\left(\sum_{\alpha \in G} \boldsymbol{\varphi}_{\alpha} \otimes \varepsilon_{\alpha}\right)=\sum_{\alpha \in G} u_{\sigma} \boldsymbol{\varphi}_{\alpha} \otimes \varepsilon_{\sigma \alpha}
$$

for all $\sum_{\alpha \in G} \boldsymbol{\varphi}_{\alpha} \otimes \varepsilon_{\alpha} \in \mathbf{H} \otimes l_{2}(G)$. The set of all operators $a \otimes 1(a \in \mathbf{M})$ will be denoted by $\mathbf{M} \otimes \mathbf{I}$. For $A=a \otimes 1 \in \mathbf{M} \otimes \mathbf{I}$ and $\sigma \in G$ we denote $a^{\sigma} \otimes 1$ by $A^{\sigma}$. Then it is clear that

$$
U_{\sigma}^{*} A U_{\sigma}=A^{\sigma} \quad \text { for all } A \in \mathbf{M} \otimes \mathbf{I} \text { and } \sigma \in G .
$$

The crossed product of $\mathbf{M}$ by $G$, denoted by $(\mathbf{M}, G)$ is the $W^{*}$-algebra on $\mathbf{H} \otimes l_{2}(G)$ generated by the set of all finite linear combinations $\sum_{i} A_{i} U_{\alpha_{t}}\left(A_{i} \in\right.$ $\left.\mathbf{M} \otimes \mathbf{I}, \alpha_{i} \in G\right)$, and $(\mathbf{M}, G)$ is of finite type. It is noted that each element $A \in(\mathbf{M}, G)$ is uniquely expressed in the form

$$
A=\sum_{\alpha \in G}^{\prime} A_{\alpha} U_{\alpha}
$$

where $A_{\alpha} \in \mathbf{M} \otimes \mathbf{I}$ and $\sum^{\prime}$ is taken in the sense of the metrical convergence, and $\phi \otimes \varepsilon_{e}$ is a separating and generating vector for the crossed product $(\mathbf{M}, G)$. The crossed product defined above seems to depend on the choice of the representation of $G$ on $\mathbf{H}$, but it is shown that the crossed product is uniquely determined by $\mathbf{M}$ and $G$ within unitary equivalence. For the details of the theory of crossed products see [4].

2. First we shall prove the following Theorem.

THEOREM 1. Let $\mathbf{M}$ be a finite factor with the invariant $C=1$ on a Hilbert space $\mathbf{H}$ and $G$ an abelian group of autornorphisms of $\mathbf{M}$. Let $\mathbf{P}$ be the fixed algebra of $G$ in $\mathbf{M}^{1}$. Then the crossed product $(\mathbf{M}, G)$ is a factor if and only if there are no $a \in \mathbf{P}(a \neq \lambda 1, \lambda$ is a scalar $)$ and $\sigma \in G(\sigma \neq e)$ such as

$$
x a=a x^{\sigma} \quad \text { for all } x \in \mathbf{M} \text {. }
$$

PROOF. Necessity. Suppose that there exist an $a \in \mathbf{P} u(a \neq \lambda 1)$ and a $\sigma \in G$ $(\sigma \neq e)$ such as $x a=a x^{\sigma}$ for all $x \in \mathbf{M}$. Since $a \in \mathbf{P}, u_{\alpha}^{*} a u_{\alpha}=a$ for all $\alpha \in G$. Thus for any $\sum_{\alpha \in G}^{\prime} X_{\alpha} U_{\alpha} \in(\mathbf{M}, G)$,

1) The fixed algebra of $G$ in $\mathbf{M}$ is the subalgebra of $\mathbf{M}$ composed ot all elements $a \in \mathbf{M}$ such that $a^{\alpha}=a$ for all $\alpha \in G$ ([3: Definition 2]). 


$$
\begin{aligned}
& \left(A U_{\sigma}^{*}\right)\left(\sum_{\alpha \in G}^{\prime} X_{\alpha} U_{\alpha}\right)=\sum_{\alpha \in G}^{\prime} A X_{\alpha}^{\sigma} U_{\sigma-1 \alpha}=\sum_{\alpha_{\epsilon} G}^{\prime} X_{\alpha} A U_{\alpha \sigma-1} \\
& =\sum_{\alpha \in G}^{\prime} X_{\alpha} A U_{\alpha}^{\prime} U_{\sigma}^{*}=\sum_{\alpha \in G}^{\prime} X_{\alpha} U_{\alpha} A U_{\sigma}^{*}=\left(\sum_{\alpha \in G}^{\prime} X_{\alpha} U_{\alpha}\right)\left(A U_{\sigma}^{*}\right)
\end{aligned}
$$

where $A=a \otimes 1$. Hence $A U_{\sigma}^{*} \in(\mathbf{M}, G) \cap(\mathbf{M}, G)^{\prime}$. On the other hand,

$$
A U_{\sigma}^{*}\left(\phi \otimes \varepsilon_{e}\right)=\dot{a} u_{\sigma}^{*} \phi \otimes \varepsilon_{\sigma-1} .
$$

where $\boldsymbol{\varphi}$ is a separating and generating trace vector for $\mathbf{M}$, and $A U_{\sigma}^{*}$ is not the scalar multiple of the identity operator on $\mathbf{H} \otimes l_{2}(G)$.

Sufficiency. Suppose that the condition in Theorem 1 is satisfied. If $\sum_{\alpha \in G}^{\prime} X_{\alpha} U_{\alpha}$ is contained in the center of $(\mathbf{M}, G)$,

$$
\left(\sum_{\alpha \in G}^{\prime} X_{\alpha} U_{\alpha}\right)\left(A U_{\sigma}\right)=\left(A U_{\sigma}\right)\left(\sum_{\alpha \in G}^{\prime} X_{\alpha} U_{\alpha}\right)
$$

for all $A=a \otimes 1 \in \mathbf{M} \otimes \mathbf{I}$ and $\sigma \in G$. Then

$$
\left(\sum_{\alpha \in G}^{\prime} X_{\alpha} U_{\alpha}\right)\left(A U_{\sigma}\right)=\sum_{\alpha \in G}^{\prime} X_{\alpha} A^{\alpha-1} U_{\alpha \sigma}
$$

and

$$
\left(A U_{\sigma}\right)\left(\sum_{\alpha \in G}^{\prime} X_{\alpha} U_{\alpha}\right)=\sum_{\alpha \in G}^{\prime} A X_{\alpha}^{\sigma-1} U_{\alpha \sigma} .
$$

Thus we have $a x_{\alpha}^{\sigma-1}=x a_{\alpha}^{\alpha-1}$ for each $\alpha \in G$ where $X_{\alpha}=x_{\alpha} \otimes 1$. Take $\sigma=e$, and we have

$$
a x_{\alpha}=x_{\alpha} a^{\alpha-1} \quad \text { for all } a \in \mathbf{M} .
$$

Hence by the assumption $x_{\alpha}=\lambda_{\alpha} 1$ for all $\alpha \in G, \alpha \neq e$ where $\lambda_{\alpha}$ are scalars, and so $a=a^{\alpha-1}$ for all $\alpha \in G, \alpha \neq e$ if $\lambda_{x} \neq 0$ for $\alpha \neq e$, which contradicts to the arbitrariness of $a \in \mathbf{M}$. From the relation $a x_{\alpha}=x_{\alpha} a^{\alpha^{-1}}$ for all $a \in \mathbf{M}$, $x_{e}$ is the scalar multiple of the identity operator on $\mathbf{H}$, and $(\mathbf{M}, G)$ is a factor.

As a corollary of Theorem 1, we obtain the slight improvement of the example in [4]:

COROLLARY 1. Let $\mathbf{M}$ be a finite factor with the invariant $C=1$ on a Hilbert space and $\alpha$ a non-trivial automorphism of $\mathbf{M}$. Let $G$ be a cyclic group generated by $\alpha$. Then $\alpha$ is outer if the crossed product $(\mathbf{M}, G)$ is a factor. In particular, when the order of $\alpha$ is 2 or $3,(\mathbf{M}, G)$ is a factor if and only if $G$ is outer.

PROOF. Suppose that $(\mathbf{M}, G)$ is a factor and $\boldsymbol{\alpha}$ is inner. Then there exists a unitary operator $u \in \mathbf{M}$ such that $u^{*} a u=a^{\alpha}$ for all $a \in \mathbf{M}$. Since $(u)^{\alpha^{n}}=$ $\left(u^{*}\right)^{n} u(u)^{n}=u$ for all $n=0, \pm 1, \pm 2, \ldots, u$ is contained in the fixed algebra of $G$ in M. Moreover we have 


$$
a u=u a^{\alpha} \quad \text { for all } a \in \mathbf{M} \text {. }
$$

Thus, by Theorem $1,(\mathbf{M}, G)$ is not a factor which is a contradiction, and $\alpha$ is an outer automorphism of $\mathbf{M}$.

To prove the second part of our assertion, it is sufficient to show the "only if" part because the "if part" is known ([1], [4]). Suppose that $(\mathbf{M}, G)$ is a factor and $\alpha^{3}=e . \alpha$ is an outer automorphism of $\mathbf{M}$ as shown above, and since $\alpha^{2}=\alpha^{-1}, \alpha^{2}$ is also an outer automorphism of $\mathbf{M}$. Thus $G$ is outer. If $\alpha^{2}=e$, it is obvious that $G$ is outer.

The case where $\alpha^{2}=e$ is nothing but the example in [3].

By virtue of Corollary 1 we can prove the following Theorem which is closely related to [2] and sharpens the results in [2].

THEOREM 2. Let $\mathbf{M}$ and $\mathbf{N}$ be finite factors with the invariants $C=1$, and let $G$ and $H$ be groups of outer automorphisms of $\mathbf{M}$ and $\mathbf{N}$ respectively. Then $G \times H^{2)}$ is a group of outer automorphisms of $\mathbf{M} \otimes \mathbf{N}$.

PRoOF. Let $(\alpha, \beta) \in G \times H$ be an arbitrary element which is different from the unit $(e, e)$ of $G \times H$, and let $\mathfrak{G}_{(\alpha, \beta)}$ be a cyclic group generated by $(\alpha, \beta)$. Then it is sufficient to show that the crossed product $\left(\mathbf{M} \otimes \mathbf{N}, \mathbb{S}_{(\alpha, \beta)}\right)$ is a factor by Corollary 1. By [2: Theorem 1] and [3: Theorem] we have 1

$$
\begin{aligned}
\left(\mathbf{M} \otimes \mathbf{N}, \mathbb{S}_{(\alpha, \beta)}\right) \cap\left(\mathbf{M} \otimes \mathbf{N}, \mathbb{S}_{(\alpha, \beta)}\right)^{\prime} \subseteq(\mathbf{M} \otimes \mathbf{N}, G \times H) \cap(\mathbf{M} \otimes \mathbf{N},\{(e, e)\})^{\prime} \\
=(\mathbf{M}, G) \otimes(\mathbf{N}, H) \cap((\mathbf{M},\{e\}) \otimes(\mathbf{N},\{e\}))^{\prime} \\
=\left((\mathbf{M}, G) \cap(\mathbf{M},\{e\})^{\prime}\right) \otimes\left((\mathbf{N}, H) \cap(\mathbf{N},\{e\})^{\prime}\right) .
\end{aligned}
$$

On the other hand by [4: Theorem 3], $(\mathbf{M}, G) \cap(\mathbf{M},\{e\})^{\prime}\left(\operatorname{resp} .(\mathbf{N}, H) \cap(\mathbf{N},\{e\})^{\prime}\right)$ coincides with the center of $(\mathbf{M},\{\boldsymbol{e}\})(\operatorname{resp} .(\mathbf{N},\{\boldsymbol{e}\}))$, because $G(\operatorname{resp} . H)$ is outer. Thus $\left(\mathbf{M} \otimes \mathbf{N}, \mathfrak{S}_{(\alpha, \beta)}\right)$ is a factor, and the proof is completed.

REMARK. Theorem 2 holds when $\mathbf{M}$ and $\mathbf{N}$ are semi-finite factors. A sketch of the proof is as follows. Let $\mathbf{M}$ be a standard factor on a Hilbert space $\mathbf{H}$ and $G$ a group of automorphisms of $\mathbf{M}$. Then Lemmas 1 and 2 in [4] remain true, and so we can define the crossed product as the same way as in the case of finite factor, and Lemma 5 and Theorem 3 in [4] are also true ${ }^{3}$. Hence we can easily seen that Corollary 1 is valid and the same computations as the proof of Theorem 2 are available.

3. Let $\mathbf{M}$ be a finite factor with the invariant $C=1$ on a Hilbert space $\mathbf{H}$ and $G$ a group of automorphisms of $\mathbf{M}$. Let $\mathbf{P}$ be the fixed algebra of $G$ in

2) For the definition of $G \times H$, see Lemma 2 in [2].

3) These facts were pointed out by N. Suzuki when he published the paper [4]. 
M. Then $(\mathbf{P}, G)$ means the $W^{*}$-subalgebra of the crossed product $(\mathbf{M}, G)$ generated by all finite linear combinations $\sum_{i} A_{i} U_{\alpha_{i}}$ where $A_{i}=a_{i} \otimes 1, \alpha_{i} \in G$. It is easily seen that each element in $(\mathbf{P}, G)$ can be expressed uniquely in the form $\sum_{\alpha \in G} A_{\alpha} U_{\alpha}$ where $A_{\alpha}=a_{\alpha} \otimes 1, a_{\alpha} \in \mathbf{P}$. The set of all operators $a \otimes 1$ on $\mathbf{H} \otimes l_{2}(G)$ such as $a \in \mathbf{P}$ will be denoted by $\mathbf{P} \otimes \mathbf{I}$.

LEMMA 1. If $G$ is abelian

$$
(\mathbf{P}, G)=(\mathbf{M}, G) \cap R\left(U_{\alpha} \mid \alpha \in G\right)^{\prime} .
$$

PROOF. We first recall that $a \in \mathbf{P}$ if and only if $a u_{\alpha}=u_{\alpha} a$ for all $\alpha \in G$. Let $\sum_{a \in G}^{\prime} A_{\alpha} U_{a}$ be an element in $(\mathbf{P}, G)$. Then for each $\sigma \in G$ we have

$$
\begin{gathered}
U_{\sigma}\left(\sum_{\alpha \in G}^{\prime} A_{\alpha} U_{\alpha}\right)=\sum_{\alpha \in G}^{\prime} U_{\sigma} A_{\alpha} U_{\alpha}=\sum_{\alpha \in G}^{\prime} A_{\alpha} U_{\sigma \alpha} \\
=\sum_{\alpha \in G}^{\prime} A_{\alpha} U_{\alpha \sigma}=\left(\sum_{\alpha \in G}^{\prime} A_{\alpha} U_{\alpha}\right) U_{\sigma},
\end{gathered}
$$

and so

$$
(\mathbf{P}, G) \subseteq(\mathbf{M}, G) \cap R\left(U_{\alpha} \mid \alpha \in G\right)^{\prime}
$$

Conversely, if we take an arbitrary element $\sum_{\alpha \in G}^{\prime} A_{\alpha} U_{\alpha}$ in (M. $\left.G\right) \cap$ $R\left(U_{\alpha} \mid \alpha \in G\right)^{\prime}$,

$$
U_{\sigma}\left(\sum_{\alpha \in G}^{\prime} A_{\alpha} U_{\alpha}\right)=\left(\sum_{\alpha \in G}^{\prime} A_{\alpha} U_{\alpha}\right) U_{\sigma} \text { for all } \sigma \in G .
$$

Thus

$$
\sum_{\alpha_{\epsilon} G \dot{x}}^{\prime} A_{\alpha}^{\sigma-1} U_{\alpha_{\sigma}}=\sum_{\alpha_{\epsilon \in}}^{\prime} A_{\alpha} U_{\alpha \sigma} \text { for all } \sigma \in G,
$$

hence we have $a_{\alpha}=a_{\alpha}^{\sigma-1}$ for each $\alpha \in G$ where $A_{\alpha}=a_{\alpha} \otimes 1 \in \mathbf{M} \otimes \mathbf{I}$. Since $\sigma \in G$ is arbitrary, $a_{\alpha} \in \mathbf{P}$ for all $\alpha \in G$. This proves that $\sum_{\alpha \in G}^{\prime} A_{\alpha} U_{\alpha} \in$ $(\mathbf{P}, G)$ and

$$
(\mathbf{P}, G) \supseteqq(\mathbf{M}, G) \cap R\left(U_{\alpha} \mid \alpha \in G\right)^{\prime},
$$

So we have $(\mathbf{P}, G)=(\mathbf{M}, G) \cap R\left(U_{\alpha} \mid \alpha \in G\right)^{\prime}$.

As an immediate consequence of Lemma 1, we have the following result.

COROLLARY 2. If $G$ is ergodic and abelian, $R\left(U_{\alpha} \mid \alpha \in G\right)$ is a maximal abelian $W^{*}$-subalgebra of the factor $(\mathbf{M}, G)$.

In fact, the ergodicity of $G$ leads to $\mathbf{P}=\{\lambda 1\}$, and $(\mathbf{P}, G)=R\left(U_{\alpha} \mid \alpha \in G\right)$. Thus, by Lemma 1 ,

$$
R\left(U_{\alpha} \mid \alpha \in G\right)=(\mathbf{M}, G) \cap R\left(U_{\alpha} \mid \alpha \in G\right)^{\prime} .
$$


This proves Corollary 2 since $R\left(U_{\alpha} \mid \alpha \in G\right)$ is abelian.

Next lemma is a non-abelian analogue of Lemma 1.

LEMMA 2. Assume that $G$ satisfies the condition: every non-trivial conjugate class of $G$ it infinite, that is for every $\alpha \in G$ other than the identity, the class $\left\{\sigma \alpha \sigma^{-1} \mid \sigma \in G\right\}$ is infinite. Then we have

$$
(\mathbf{M}, G) \cap R\left(U_{\alpha} \mid \alpha \in G\right)^{\prime}=\mathbf{P} \otimes \mathbf{I} \text {. }
$$

PROOF. Let $A=\sum_{\alpha \in G}^{\prime} A_{\alpha} U_{\alpha}$ be an arbitrary element in $(\mathbf{M}, G) \cap R\left(U_{\alpha} \mid \alpha \in G\right)^{\prime}$. Then

$$
A U_{\sigma}=U_{\sigma} A \quad \text { for all } \sigma \in G .
$$

Since $A U_{\sigma}=\sum_{\alpha G}^{\prime} A_{\alpha} U_{\alpha \sigma}=\sum_{\alpha^{\alpha} G}^{\prime} A_{\sigma \alpha \sigma-1} U_{\sigma \alpha}$ and $U_{\sigma} A=\sum_{\alpha \in G}^{\prime} A_{\alpha}^{\sigma-1} U_{\sigma \alpha}$, we have

$$
a_{\alpha}^{\sigma-1}=a_{\sigma \alpha \sigma-1} \quad \text { for all } \sigma \in G \text { and } \alpha \in G,
$$

where $A_{\alpha}=a_{\alpha} \otimes 1 \in \mathbf{M} \otimes \mathbf{I}$. Suppose that $a_{\alpha_{0}} \neq 0$ for an $\alpha_{0} \in G, \alpha_{0} \neq e$. Let $\boldsymbol{\varphi}$ be a separating and generating trace vector for $\mathbf{M}$. Then, by our hypothesis the conjugate class $\left\{\sigma \alpha_{0} \sigma^{-1} \mid \sigma \in G\right\}$ is infinite. As $\|a \varphi\|=\left\|a^{\sigma} \phi\right\|$ for all $a \in \mathbf{M}$ and $\sigma \in G$, we have by $\left({ }^{*}\right)$

$$
\left\|a_{\sigma \alpha_{0} \sigma-1} \varphi\right\|=\left\|a_{\alpha_{0}}^{\sigma-1} \varphi\right\|=\left\|a_{\alpha_{0}} \boldsymbol{\phi}\right\| \text { for all } \sigma \in G .
$$

Thus we have

$$
\sum_{\alpha \in G}^{\prime}\left\|a_{\alpha} \boldsymbol{\varphi}\right\|^{2}=\infty \text {. }
$$

which is a contradiction. Hence $a_{\alpha}=0$ for. all $\alpha \in G, \alpha \neq e$, and $A=A_{e} \in \mathbf{P} \otimes \mathbf{I}$ because, again by $\left(^{*}\right) a_{e}^{\sigma-1}=a_{e}$ for all $\boldsymbol{\sigma} \in G$, and so $(\mathbf{M}, G) \cap R\left(U_{\alpha} \mid \alpha \in G\right)^{\prime}$ $\sqsubseteq \mathbf{P} \otimes \mathbf{I}$. On the other hand, it is obvious that $\mathbf{P} \otimes \mathbf{I} \leqq(\mathbf{M}, G) \cap R\left(U_{\alpha} \mid \alpha \in G\right)^{\prime}$ since $a u_{\alpha}=u_{\alpha} a$ for all $a \in \mathbf{P}$ and $\alpha \in G$. Therefore we have

$$
(\mathbf{M}, G) \cap R\left(U_{\alpha} \mid \alpha \in G\right)^{\prime}=\mathbf{P} \otimes \mathbf{I} .
$$

By Lemma 2 we have the following theorem.

THEOREM 3. Let $\mathbf{M}$ be a finite factor with the invariant $C=1$ and $G$ a group of automorphisms of $\mathbf{M}$ whose non-trivial conjugate classes are all infinite. Then $(\mathbf{M}, G)$ is a factor.

PROOF. Let $A=\sum_{\alpha \in G}^{\prime} A_{\alpha} U_{\alpha}$ be an arbitrary element in the center of $(\mathbf{M}, G)$. Since $(\mathbf{M}, G) \cap(\mathbf{M}, G)^{\prime} \leqq(\mathbf{M}, G) \cap R\left(U_{\alpha} \mid \alpha \in G\right)^{\prime}, A=A_{e} \in \mathbf{P} \otimes \mathbf{I}$, where $\mathbf{P}$ is the fixed algebra of $G$ in $\mathbf{M}$ by Lemma 2. Moreover $A$ commutes with all $x \otimes 1 \in \mathbf{M} \otimes \mathbf{I}$, and so we have $a_{e} x=x a_{e}$ for all $x \in \mathbf{M}$ where $A_{e}=a_{e} \otimes 1$. Thus $a_{e} \in \mathbf{M} \cap \mathbf{M}^{\prime}$. Hence $A$ is the scalar multiple of the identity operator on 
$\mathbf{H} \otimes l_{2}(G)$, and $(\mathbf{M}, G)$ is a factor.

REMARK. Theorem 3 can be slightly generalized as follows. Assume that $G$ has a subgroup $G_{0}$ such that for every element $\alpha \in G$ other than the identity, the set $\left\{\sigma \alpha \sigma^{-1} \mid \sigma \in G_{0}\right\}$ is infinite. Then the commutant of $\left(\mathbf{M}, G_{0}\right)$ in the crossed product $(\mathbf{M}, G)$ is the scalar multiples of the identity operator on $\mathbf{H} \otimes l_{2}(G)$, where $\left(\mathbf{M}, G_{0}\right)$ is a subalgebra of $(\mathbf{M}, G)$ composed of all $A$ $=\sum_{\alpha \in G_{0}}^{\prime} A_{\alpha} U_{\alpha} \in(\mathbf{M}, G)$. In particular $(\mathbf{M}, G)$ is a factor.

This is a non-commutative version of Lemma 3 in [5].

\section{REFERENCES}

[1] T.SAITo, The direct product and the crossed product of rings of opdrators, Tôhoku Math. Journ. 11(1959), 229-304.

[2] T.SAItô and J. TOMiY AmA, Some results on the direct product of $W^{*}$-algebras, Tôhoku Math. Journ., 12(1960), 455-458.

[3] N.SUZUKI, Crossed products of rings of operators, Tôhoku Math. Journ., 11(1959), 113-124.

[4] H. WIDOM, Nonisomorphic approximately finite factors. Proc. Amer. Math. Soc., 8(1957), 537-540.

MATHEMATICAl INSTituTe, TôHOKU UNiversity. 